\title{
Experimental research of solid waste drying in the process of thermal processing
}

\author{
Vyacheslav V. Bukhmirov, Olga B. Kolibaba, Ramil N. Gabitov \\ Ivanovo State Power Engineering University, 153003 Ivanovo, Russia
}

\begin{abstract}
The convective drying process of municipal solid waste layer as a polydispersed multicomponent porous structure is studied. On the base of the experimental data criterial equations for calculating heat transfer and mass transfer processes in the layer, depending on the humidity of the material, the speed of the drying agent and the layer height are obtained. These solutions are used in the thermal design of reactors for the thermal processing of multicomponent organic waste.
\end{abstract}

\section{INTRODUCTION}

Thermal methods, which include pyrolysis and gasification are widespread waste technologies. Thermal processing of raw materials, makes it possible to obtain not only the combustible gas, but also to solve the environmental problems associated with urban pollution and land reclamation.

The process of thermal processing of solid waste is most often carried out in shaft furnaces (thermal reactors) [1,2]. Municipal solid waste is loaded into the upper part of the reactor and consistently held drying, pyrolysis and gasification zones.

The drying zone is carried out heating and drying of solid waste layer by heat exchange with a stream of hectares of the area downstream from the pyrolysis, and allocated in conjunction with the moisture of light-volatile compounds is supplied to the gasification of solid carbonaceous residues (SCR).

In the pyrolysis zone the combustible components of MSW pyrolyzed to form the pyrolysis gas, a liquid product comprised of polyaromatic hydrocarbons, and SCR. Solid carbonaceous residue, gradually falling to the bottom of the reactor, feds to the gasification zone. There also oxidant gas (steam mixture) is directed. The resulting SCR gasification fuel gas in a mixture with the pyrolysis gas is a fuel quality suitable for use.

Solid waste can be attributed to particulate material, which in the presence of moisture internal require high energy consumption for the drying process. In order to select a rational regime of the reactor it is necessary to determine the temperature field of solid waste layer, drying time raw material and energy costs in the process. Knowledge of these parameters is possible to calculate the speed of charging, the height of the drying zone of the thermal reactor and, therefore, to design it properly.

\section{Problem statement}

For the convective drying conjugate problem of heat and mass transfer, which is characterized by the following is solved:

- drying agent gives heat to the dried up material and takes the moisture evaporates;

- drying material receives the heat of the drying agent and gives it moisture.

The drying agent is used as the transport means. 
In drying theory, the method of the analysis of external heat and mass transfer [4], based on the joint consideration of the equations of motion and continuity of viscous incompressible flow:

$$
\begin{gathered}
\frac{\partial \vec{v}}{\partial \tau}+\vec{v} \cdot \operatorname{grad} \vec{v}=\vec{g}-\frac{1}{\rho} \operatorname{grad} P+v \nabla^{2} \vec{v} \\
\frac{\partial \rho}{\partial \tau}+\operatorname{div} \rho \vec{v}=0
\end{gathered}
$$

convection-diffusion transport of moisture in a moving drying agent:

$$
\frac{\partial C}{\partial \tau}+\vec{v} \cdot \operatorname{grad} C=D \nabla^{2} C
$$

and the equation describing the temperature field in the flow of coolant is used:

$$
\frac{\partial t}{\partial \tau}+\vec{v} \cdot \operatorname{grad} t=a \nabla^{2} t
$$

$v$ - speed of the drying agent, $\mathrm{m} / \mathrm{s} ; \mathrm{g}$ - gravitational acceleration, $\mathrm{m} / \mathrm{s}^{2} ; \rho$ - the density of the drying agent, $\mathrm{kg} / \mathrm{m}^{3} ; \mathrm{P}$ - pressure, $\mathrm{Pa} ; \mathrm{v}$ - kinematic viscosity coefficient, $\mathrm{m}^{2} / \mathrm{s} ; \mathrm{C}$ - moisture concentration, $\mathrm{m}^{3}$ $/ \mathrm{m}^{3} ; \mathrm{D}$ - molecular diffusion coefficient, $\mathrm{m}^{2} / \mathrm{s} ; \mathrm{t}$ - temperature, ${ }^{\circ} \mathrm{C}$; a - coolant thermal diffusivity, $\mathrm{m} /$ $s^{2}$.

The difficulties of external heat and mass transfer analysis by the theoretical methods result in the need of a similarity theory with the using the experimental data.

\section{EXPERIMENT}

We carried out experimental studies of the convective drying process of wet solid waste layer in the four temperature conditions during gas flow rates: $0.1,0.5,1,1.5,2 \mathrm{~m} / \mathrm{s}$. The first mode of drying is carried out at a constant temperature of the drying agent, $107^{\circ} \mathrm{C}$. The second, third and fourth - at temperatures of $127^{\circ} \mathrm{C}, 147^{\circ} \mathrm{C}, 167^{\circ} \mathrm{C}$.

Figure 1 shows the experimental setup for study of the heat and mass transfer process between the solid waste layer and a drying agent (air). The experimental setup consisted of a pipe section (1) for retaining a metal mesh MSW layer (working zone), and the electric heater (2) for heating the drying medium (air). Supply air is a fan (4) located in front of heater. The air flow rate and its temperature is controlled by means of the control unit (3) with a screen for displaying information. The temperature and the humidity was recorded by sensors RHP-2S11 (5) readout on the screen of the control unit. The air flow rate was measured by an anemometer Testo 416 (6). During the experiment, the temperature of the sample was measured 5 thermocouples TCA established in the layer of solid waste. Analog-todigital converter of the signal OWEN MVA8 transmited a signal to a personal computer. Drying of the sample continued to establish its constant weight.

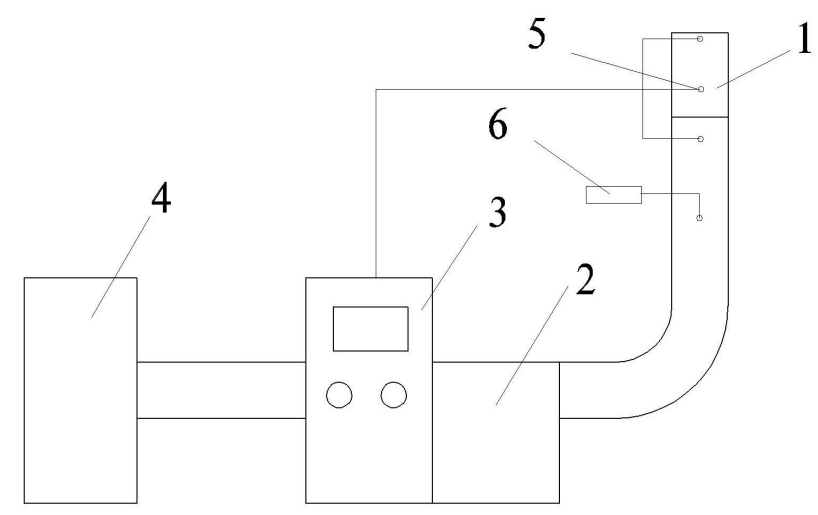

Figure 1. Experimental installation for the investigation of solid waste drying processes: 1 Operating zone; 2 - Electric heater; 3 - Control block; 4 - Fan; 5 - The temperature sensors; 6 Anemometr 
Before experiment samples of municipal solid waste average morphological composition were placed in a water bath for 20 hours. With the help of centrifuge the excess moisture in samples is removed. Then the MSW sample is placed in the installation working zone. To determine the moisture content of MSW sample weighed portion was recovered. It was weighed on electronic scales laboratory (VL E134) 4th grade accuracy, placed in an oven heated to a temperature of $107^{\circ} \mathrm{C}$, and kept up-achievements of constant weight.

During the drying process the change in mass of the material was captured (Fig.2). Then the calculation of humidity of raw materials and the rate of change of moisture content is carry out.

\section{Results and discussion}

Fig. 2 shows experimental data of temperature change MSW layer during its drying, and change in humidity sample at four temperature conditions of the drying agent, at a speed of $0.1 \mathrm{~m} / \mathrm{s}$.

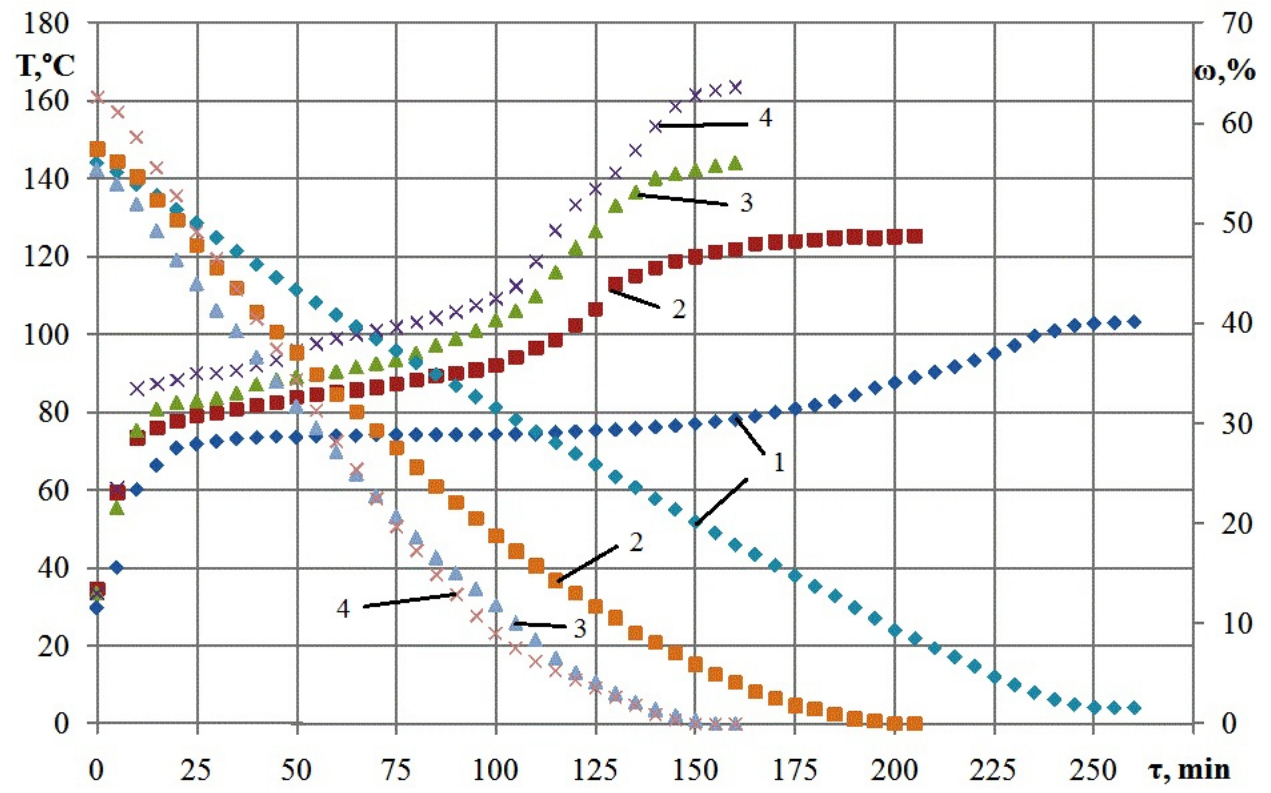

Figure 2. The temperature and humidity of the sample MSW layer in the drying process at four temperature regimes of the drying agent

$$
\left(1-107^{\circ} \mathrm{C}, 2-127^{\circ} \mathrm{C}, 3-147^{\circ} \mathrm{C} 4-167^{\circ} \mathrm{C}\right) \text { at a speed of } 0.1 \mathrm{~m} / \mathrm{s}
$$

The experimental data obtained in the process of convective drying of wet MSW layer at temperature regimes of drying agent: $107^{\circ} \mathrm{C}, 127^{\circ} \mathrm{C}, 147^{\circ} \mathrm{C}, 167^{\circ} \mathrm{C}$ and at gas flow rates: 0.1, 0.5, $1,1.5,2 \mathrm{~m} / \mathrm{s}$, it was approximated as a criteria equation:

$$
N u=A\left(\frac{d}{H}\right)^{k} \operatorname{Re}^{n} E^{m}
$$

where $\mathrm{H}$ - the height of the layer, $\mathrm{m}$; $\mathrm{d}$ - equivalent to the size; $\mathrm{E}$ - simplex, taking into account the change in moisture content (MSW), i.e, the impact of mass transfer on heat transfer.

$$
E=\frac{1-\omega}{\omega}
$$


where $\omega$ - integrated relative humidity of the material.

Calculation of mass transfer can be reduced to the calculation of heat transfer by using the ratio [3]:

$$
N u=S h \cdot K_{c}
$$

where $\mathrm{Sh}$ - Sherwood criterion; $\mathrm{K}_{\mathrm{c}}$ - correction factor for the diffusion flux.

$$
K_{c}=\frac{1}{1-p^{c p}}
$$

where $\mathrm{p}^{\mathrm{cp}}$ - mean log vapor pressure in the boundary layer.

After processing of the experimental data criterial equations for determination the coefficients of heat transfer and mass transfer were obtained.

Criteria equation for laminar:

$$
\begin{aligned}
& N u=1,24\left(\frac{d}{H}\right)^{0.3} R e^{0.33} E^{0.084}, \\
& S h=0,911\left(\frac{d}{H}\right)^{-0.2} R e^{0.33} E^{0.084}
\end{aligned}
$$

Criteria equation for turbulent regime:

$$
\begin{aligned}
& N u=0,107\left(\frac{d}{H}\right)^{0.38} \operatorname{Re}^{0.82} E^{0.12} \\
& S h=0,066\left(\frac{d}{H}\right)^{-0.49} \operatorname{Re}^{0,82} E^{0.12}
\end{aligned}
$$

In the case of heat exchange for defining the size the equivalent diameter of the piece found by direct measurement of samples with the Gaussian distribution is adopted. In the case of mass transfer the length of the evaporation surface, i.e. the minimum length of the pore canal in the direction of movement of the drying agent. For defining the temperature the average temperature of the drying agent is accepted.

Fig. 3 and 4 shows the average heat transfer coefficients of the drying agent to the material and the mass transfer in the drying MSW layer at different speeds of the drying agent. Analysis of the graphs shows that with a decrease in the relative humidity of the material intensity of the heat and mass transfer is reduced.

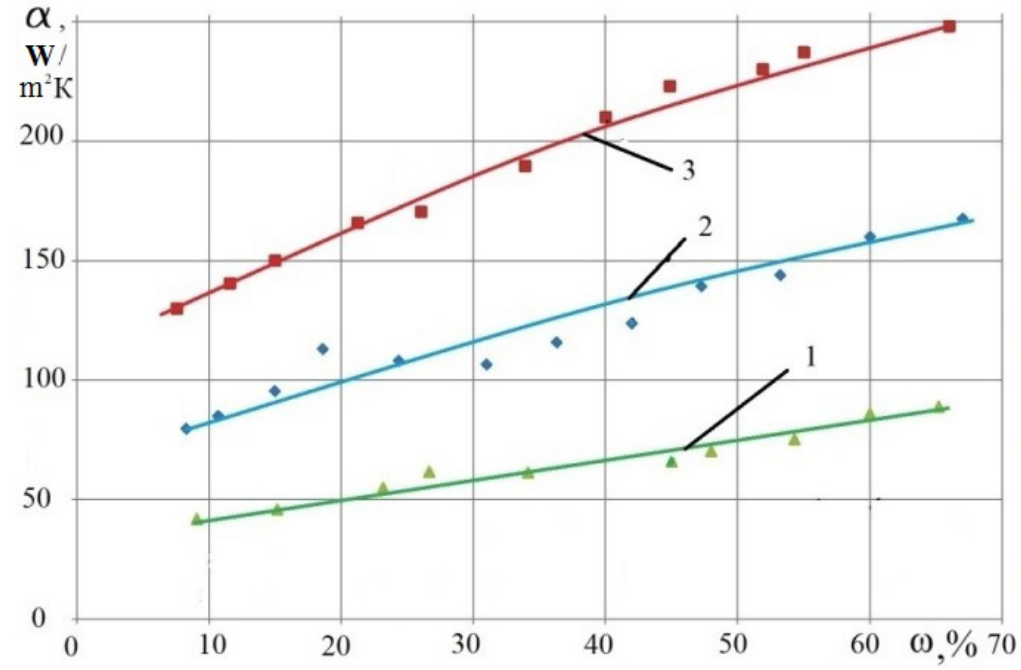

Figure 3. The dependence of the heat-transfer coefficient from the humidity during the drying process at a constant temperature of $107^{\circ} \mathrm{C}$ and the air velocity: $1-1 \mathrm{~m} / \mathrm{s} 2-1.5 \mathrm{~m} / \mathrm{s}, 3-2 \mathrm{~m} / \mathrm{s}$. 


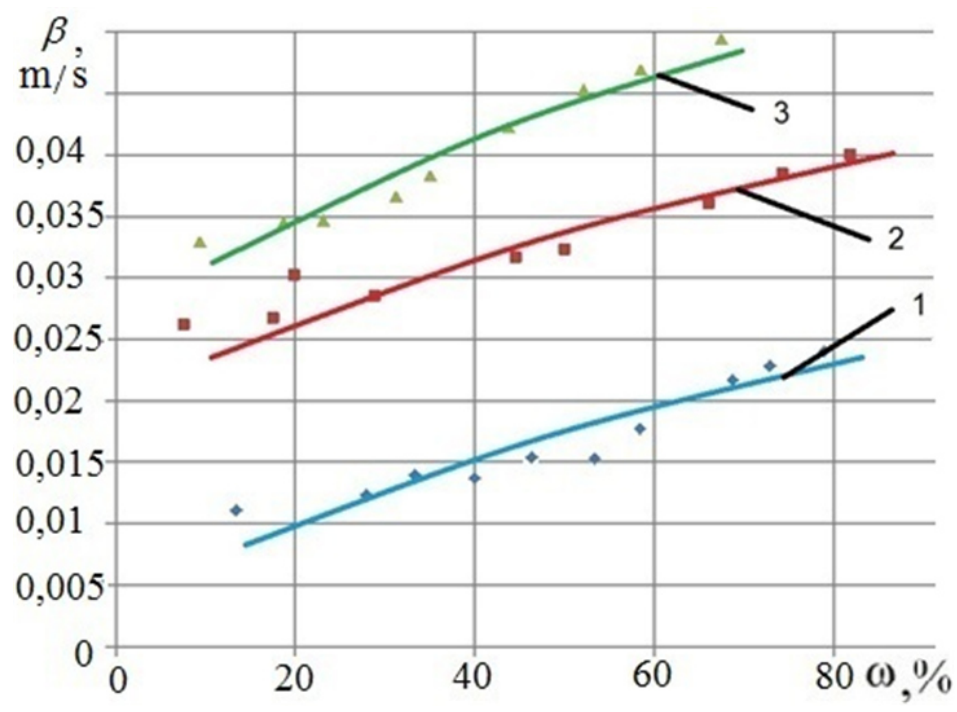

Figure 4. The dependence of the mass-transfer coefficient on the relative humidity during the drying process at a constant temperature of $107^{\circ} \mathrm{C}$ and the air velocity: $1-1 \mathrm{~m} / \mathrm{s} 2-1.5 \mathrm{~m} / \mathrm{s}, 3-2 \mathrm{~m} / \mathrm{s}$.

Processing results obtained using the setup (Fig. 1) established dependencies (Fig. 3) of the ignition delay time of coal particles with various sizes from the temperature of the heating surface.

\section{Conclusion}

An experimental study of the organic raw materials convective drying process, modeling layer of municipal solid waste, was fulfilled. As the result criterial equations for calculating heat transfer and mass transfer processes in the porous layer, depending on the humidity of the material, the speed of the drying agent, the layer height were obtained.

\section{References}

1. Dolinin D A, Gabitov R N, Semin E S, Gorinov O I, Kolibaba O B, Gorinov P O, Samyshina O V, 2014 J. Bulleten №23 (The Russian Federation patent №2525558)

2. Dolinin D A, Gorinov P O, Semin E S, Gorinov O I, Kolibaba O B, Gabitov R N, Samyshina O V, 2014 J. Bulleten №36 (The Russian Federation patent №2536896)

3. Lykov M V 1970 Drying in the chemical industry Moscow: Chemistry p 430

4. Sokolsky A I 2005 Heat treatment of disperse materials in devices with vortex two-phase flows Ivanovo: ISABU p 320 